\title{
COMPACT DYNAMICAL SYSTEMS
}

\author{
RONALD A. KNIGHT
}

\begin{abstract}
Compact flows on locally compact phase spaces are characterized in terms of the bilateral stability properties of the orbits and a stability theorem for cycles is formulated.
\end{abstract}

1. Introduction. A characterization of compact flows on dichotomic 2-manifolds in terms of the bilateral stability properties of the orbits was given by Knight in [5]. Our aim is to obtain such a characterization on a locally compact carrier. The Cycle Stability Theorem [4, p. 196] and Ura's Stability Theorem [2, Theorem 7.6] are fundamental tools used in [5]. The Cycle Stability Theorem applies only to certain 2-manifolds, consequently, we combine Ura's Alternatives [2, Theorem 9.5] with our Lemma 1 obtaining a cycle stability theorem for locally compact phase spaces.

Henceforth, we shall let $(X, \pi)$ be a given flow on a Hausdorff phase space $X$. The periodic set and the critical set are denoted by $P$ and $S$, respectively. The extension of a flow $(X, \pi)$ to the one point compactification $X^{*}$ is denoted by $\left(X^{*}, \pi^{*}\right)$. The extension of a function $F$ on $X$ is denoted by $F^{*}$ and the critical set of $\left(X^{*}, \pi^{*}\right)$ is denoted by $S^{*}$. We denote the orbit through $x$, orbit closure of $x$, limit set of $x$, and prolongation of $x$ by $C(x), K(x), L(x)$, and $D(x)$, respectively. By $A(M)$ we mean the region of bilateral attraction for a set $M$ in $X$. The positive and negative versions of these concepts carry the appropriate + or - superscript. The reader is referred to [1] and [6] for standard definitions and notations used herein.

A flow is called compact whenever each of its orbits is compact. An orbit is compact if and only if it is periodic or critical $[3,3.09]$. We say that a flow is of characteristic 0 on a set $M$ if and only if $D(x)=K(x)$ for each $x \in M$. A flow is of characteristic 0 if it is of characteristic 0 on the phase space. The unilateral versions of this notion are defined similarly and carry the appropriate superscript.

2. Stability of cycles. We first obtain a lemma crucial to our cycle stability theorem. The lemma would be immediate if the periodic orbit conjecture held, however, an example of a flow on a compact 5-manifold with only periodic orbits which have unbounded periods was given by Sullivan [7].

\footnotetext{
Received by the editors November 17, 1977 and, in revised form, April 26, 1978. AMS (MOS) subject classifications (1970). Primary 34C35; Secondary 54H20.

Key words and phrases. Attraction, bilateral stability, characteristic 0, compact flow, dynamical system, flow, stability.

${ }^{1}$ This research was partially supported by an NMSU faculty research grant.
} 
LEMMA 1. If there are no critical points in a flow on a locally compact space, then the flow is of characteristic 0.

Proof. Let $(X, \pi)$ be compact, $X$ be locally compact, and the critical set $S$ be empty. Then $X=P[3,3.09]$. We proceed by contradiction showing that $D(x)=C(x)$ for each $x$ in $P$. For some $x$ in $P$ let $y$ be a point of $D(x)-C(x)$. Select an open neighborhood $V$ of $C(x)$ with compact closure contained in $X-C(y)$. Let $A=V R \cap(X-\bar{V}) R$ and $D=X-A$. Then $C(x) \cup C(y) \subset D, A$ is open, and $D \cap \partial V$ is compact. Define $t_{z}=\inf \{t \in$ $\left.R^{+}: z t \in V\right\}$ for $z \in A$. Evidently, $t_{z}<+\infty$. For any compact set $M \subset \partial V$ $-D$ define $T_{M}=\sup \left\{t_{z}: z \in M\right\}$. For $z \in M$ there is a $z t \in V$ where $0<t<t_{z}+1$. The continuity of $\pi$ yields $t_{p} \leqslant t<t_{z}+1$ for each $p$ in some neighborhood $V_{z}$ of $z$. There is a finite subcover $\left\{V_{z_{1}}, \ldots, V_{z_{k}}\right\}$ of $M$. For each $p \in \cup\left\{V_{z_{i}}: 1 \leqslant i \leqslant k\right\}$ we have $t_{p} \leqslant \max \left\{t_{z_{i}}+1: 1 \leqslant i \leqslant k\right\}$ so that $T_{M}<+\infty$. Next, let $\left(x_{i}\right)$ be a net in $V$ converging to $x$ and let $\left(r_{i}\right)$ be a net in $R^{+}$such that $x_{i} r_{i} \rightarrow y$. There is a net $\left(x_{n_{i}} \tau_{n_{i}}\right)$ in $\partial V$ converging to a point $z$ in $\partial V$ where $\left(x_{n_{i}}\right)$ is a subnet of $\left(x_{i}\right)$. Choose $\tau_{n_{i}}$ to be the maximum value of $R^{+}$ in $\left[0, r_{n_{i}}\right]$ such that $x_{n_{i}} \tau_{n_{i}}$ is in $\partial V$. First, suppose that $z \in \partial V-D$. There is a compact neighborhood $G$ of $z$ disjoint from $D$. Let $M=G \cap \partial V$. Ultimately, $x_{n_{i}} r_{n_{i}}=\left(x_{n_{i}} \tau_{n_{i}}\right)\left(r_{n_{i}}-\tau_{n_{i}}\right) \in M\left[0, T_{M}\right]$. Thus, $y \in M\left[0, T_{M}\right]$ and $C(y)$ meets $\bar{V}$ which is absurd. We must have $z$ in $D$ rather than in $\partial V-D$. For some $t>0$ let $G$ be a neighborhood of $z t$ with compact closure such that $z \in X-$ $\bar{G}$ and $C(x) \cup C(y) \subset X-\bar{G}$. If each compact subneighborhood of $G$ contains a complete orbit, then there is a net $\left(y_{i}\right)$ in $\bar{G}$ converging to $z t$ such that $C\left(y_{i}\right) \subset \bar{G}$ for each $i$. But $z \in X-\bar{G}$ and we have $y_{i}(-t) \rightarrow z$ which is clearly impossible. Thus, choose $\bar{G}$ so that it contains no complete orbit. Now, either $C(z) \subset \bar{V}$ or $C(z) \subset X-V$ meeting both $X-\bar{V}$ and $\partial V$. First, we suppose that $C(z) \subset \bar{V}$ and let $V_{0}=V-\bar{G}$. Then $V_{0}$ is a neighborhood of $C(x)$ with compact closure excluding $C(y)$. Using notation for $V_{0}$ similar to that used above for $V$, we have $z \in \partial V_{0}-D_{0}$ so that $C(y)$ must meet $\partial V_{0}$ which is impossible. Hence, $C(z) \subset X-V$ but not $\partial V$. Let $T>0$ be the fundamental period of $z$ and let $V_{0}$ be the interior of $\bar{V}[0, T]$. Again we have a neighborhood $V_{0}$ of $C(x)$ with compact closure excluding $C(y)$. Furthermore, $\beta_{n_{i}}=\tau_{n_{i}}+T$ is the maximum value of $\left[0, r_{n_{i}}\right]$ such that $z_{n_{i}} \beta_{n_{i}}$ is in $\partial V_{0}$. We now have $z_{n_{i}} \beta_{n_{i}} \rightarrow z T=z$ in $\partial V_{0}$ where $C(z) \subset \bar{V}_{0}$. Already we have seen that this is absurd. Hence, we must conclude that $D(x)=C(x)$. The proof is complete.

According to Ura's Alternatives each periodic orbit $C(x)$ has one of the following properties. (i) $C(x)$ is asymptotically stable. (ii) $C(x)$ is negatively asymptotically stable. (iii) There exist points $y, z \notin C(x)$ such that $L^{+}(y)=$ $L^{-}(z)=C(x)$. (iv) For every neighborhood $V$ of $C(x)$ there is a complete orbit $C(y) \subset V-C(x)$.

In Lemma 1 we have shown that the orbits of $P^{0}$ satisfy only alternative (iv). Boundary orbits of $P$ may have any of the properties. Those orbits 
having property (i) or (ii) are easily identified and are unilaterally stable. According to the Weak Attractor Theorem [2, Theorem 8.20] if $C(x)$ is a periodic bilateral attractor, then either $C(x)$ is a component of $X$ or the smallest bilaterally stable set containing $C(x)$ is $D(x)=A(x)$. Moreover, any periodic orbit $C(x) \subset \partial P$ satisfying condition (iii) has $D(x) \neq C(x) \neq D^{ \pm}(x)$ so that it cannot be bilaterally or unilaterally stable. The following theorem is a partial extension of the Cycle Stability Theorem to locally compact spaces.

THEOREM 2. Let $(X, \pi)$ be a flow on a locally compact space $X$. Then

(i) each orbit of $P^{0}$ is bilaterally stable,

(ii) a periodic boundary orbit $C(x)$ of $P$ is not stable in any sense if there exist $y, z \notin C(x)$ such that $L^{+}(y)=L^{-}(z)=C(x)$, and

(iii) a periodic boundary orbit $C(x)$ of $P$ is respectively stable, negatively stable, or bilaterally stable provided that the flow is of characteristic $0^{+}, 0^{-}$, or 0 on $C(x)$.

Corollary 2.1. If $(X, \pi)$ is a compact flow on a locally compact space $X$, then each orbit of $P$ is bilaterally stable.

COROLlary 2.2. On a locally compact phase space a compact flow is of characteristic 0 if and only if each critical point is bilaterally stable.

Proposition 3. A compact flow on a locally compact space is of characteristic 0 if and only if it is of characteristic $0^{+}, 0^{-}$or $0^{ \pm}$.

Proof. In view of the fact that a compact flow on a locally compact space is of characteristic 0 if and only if its extended flow is of characteristic 0 , Corollary 6.3 of [6] applies.

3. Compact flows. The following lemma is given in [5]. The proof is sufficient for locally compact carriers provided we use Theorem 2 in place of the Cycle Stability Theorem for dichotomic 2-manifolds.

LEMMA 4. Let $(X, \pi)$ be compact and $X$ be locally compact. Each compact component of $S$ is bilaterally stable.

A flow with a compact 2-manifold minimal phase space is obtained by requiring $f(0,0)>0$ in the example of a flow on a torus described in [6]. Evidently, Theorem 4 of [5] is not valid for all 2-manifolds. Similar compact flows exist when $P \cup S$ is nonempty. A nest of concentric toral flows like the one above converging to a critical torus would be such a flow. For this reason we add an attraction criterion to Theorem 4 obtaining a characterization of compact flows for locally compact carriers.

THEOREM 5. A flow on a locally compact space $X$ is compact if and only if $S^{*}$ is bilaterally stable, each periodic orbit is bilaterally stable, and $L(X)=P \cup S$.

Proof. If the flow $(X, \pi)$ is compact, then the three conditions follow from Theorem 2 and Lemma 4 applied to $\left(X^{*}, \pi^{*}\right)$. 
Conversely, we proceed by showing that $P$ is open. For $y \in P$ select a compact invariant neighborhood $V$ of $C(y)$ disjoint from $S^{*}$. Then $K(z)$ is compact for any $z \in V$ and $\varnothing \neq L(z) \subset P$. If $z \notin P$, select a point $q \in L(z)$ and a compact invariant neighborhood $V_{0}$ of $C(q)$ excluding $z$. This leads to the absurdity $K(z) \cap C(q)=\varnothing$. Hence, $P$ is open.

Next, if $x \in X-P \cup S$, then $L^{*}(x) \subset S^{*}$ since $P$ is open. Clearly this is impossible since a compact invariant neighborhood of $S^{*}$ excluding $x$ exists. Whence, $(X, \pi)$ is compact.

COROLlary 5.1. A flow on a locally compact space $X$ is compact if and only if each component of the boundary of $S^{*}$ is bilaterally stable, each periodic orbit is bilaterally stable, and $L(X)=P \cup S$.

COROLlaRY 5.2. A closed flow on a locally compact space is compact if and only if $S^{*}$ and each periodic orbit are bilaterally stable.

THEOREM 6. A flow on a locally compact space is compact if and only if $S^{*}$ is bilaterally stable, each periodic orbit is bilaterally stable, and $P \cup S$ is a global attractor.

Proof. A slight modification of the proof of Theorem 5 is required since the attraction condition yields $A^{+}(P \cup S)=L^{+}(X)$.

COROLlaRY 6.1. A flow on a connected locally compact space is compact if and only if $S^{*}$ is bilaterally stable, each periodic orbit is bilaterally stable, and $P \cup S$ is a closed attractor.

Proof. We need only consider sufficiency of the conditions. If $x \in A^{+}(P$ $\cup S)-P \cup S$, then $L^{*}(x) \cap S^{*} \neq \varnothing$. As before this is not possible implying that $A^{+}(P \cup S)=P \cup S$ is open.

The author wishes to thank Professor Otomar Hajek and Roger McCann for their helpful communications on Lemma 1.

\section{REFERENCES}

1. S. Ahmad, Strong attraction and classification of certain continuous flows, Math. Systems Theory 5 (1971), 157-163. MR 46 \#4507.

2. N. Bhatia and O. Hajek, Local semi-dynamical systems, Lecture Notes in Math., vol. 90, Springer-Verlag, Berlin and New York, 1969. MR 40 \#4559.

3. W. Gottschalk and G. Hedlund, Topological dynamics, Amer. Math. Soc. Colloq. Publ., vol. 36, Amer. Math. Soc., Providence, R.I., 1955.

4. O. Hajek, Dynamical systems in the plane, Academic Press, New York, 1968. MR 39 \# 1767.

5. R. Knight, A characterization of certain compact flows, Proc. Amer. Math. Soc. 64 (1977), 52-54.

6. Structure and characterizations of certain continuous flows, Funkcial. Ekvac. 17 (1974), 223-230. MR 52 \# 1656.

7. D. Sullivan, A counterexample to the periodic orbit conjecture, Inst. Hautes Etudes Sci. Publ. Math. 46 (1976), 5-14.

Department of Mathematics, Northeast Missouri State University, Kirksville, MISSOURI 63501 